\title{
Case Report: ANNA and the Diagnosis of Lung Cancer
}

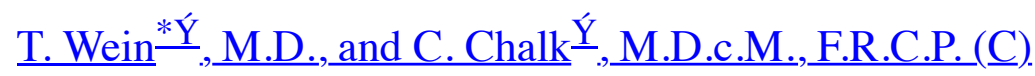

* To whom correspondence should be addressed: Ý Division of Neurology, Montreal General Hospital, McGill University, 1650 Cedar Ave., Montreal, QC, Canada H3G 1A4

\section{THE CASE}

A 60 year-old woman presented to the Peripheral Neuropathy Clinic, Montreal General Hospital, in February 1996 because of numbness in the limbs and progressive gait unsteadiness. Her symptoms began 18 months prior, when she noticed left foot numbness. Over the next two months numbness developed in her right foot and leg and 2 months, later numbness and tingling, sometimes painful, were present in all four extremities. Six months after the onset of the numbness, she noticed that her gait was unsteady. There was no history of weakness, dysautonomia, sicca symptoms, or cognitive changes.

Her past medical history was significant for 50 pack-years of smoking, migraine, and an appendectomy. Her only medicines were analgesics for migraine. The patient's father had died of gastric cancer, a brother of lung cancer, and another brother of leukemia. There was no family history of neurological disease.

Examination at another institution in 1995 was notable for areflexia, impaired joint position sense, and a wide-based gait with rombergism. A variety of blood tests (including sedimentation rate, antinuclear antibodies, rheumatoid factor, complement components $\mathrm{C} 3$ and $\mathrm{C} 4$, serum protein electrophoresis, quantitative immunoglobulins, vitamin B12, folate, and thyroid function tests), as well as chest X-ray, abdominal and pelvic ultrasound, mammography, myelography, and cranial CT scan, were normal. A sural nerve biopsy showed axonal degeneration and marked loss of myelinated nerve fibers. She was discharged home with a diagnosis of sensory neuropathy of unclear cause. A course of prednisone (30-80 mg daily) for 6 months produced no improvement.

Ten months later the patient was seen in the Peripheral Neuropathy Clinic. Her symptoms were unchanged in the preceding 6 months. Gait was wide-based and ataxic. She could only detect gross movements of the fingers and toes, and with the eyes closed and the arms outstretched there were continuous undulating movements of the fingers (pseudoathetosis). Vibratory and cool sensation were decreased in the hands and feet. Reflexes were absent. Muscle power testing was difficult because of the abnormal proprioception, but appeared normal. Nerve conduction studies showed absent sensory nerve action potentials in all limbs, whereas motor nerve conduction studies and electromyography were normal.

\section{DIFFERENTIAL DIAGNOSIS}


Clinically, the patient had an ataxic sensory neuropathy. The motor system was spared clinically and electrophysiologically, although the gross impairment of proprioception interfered with more complex motor tasks, like walking. Absent sensory nerve action potentials indicate a lesion in the sensory neuron cell bodies in dorsal root ganglia, or in their peripheral processes. As the motor fibres in the mixed peripheral nerves were normal (normal motor nerve conduction studies and electromyography), the abnormality in the sensory system was likely in the dorsal root ganglia. Anatomically, therefore, the diagnosis is a dorsal root ganglionopathy, often called a sensory neuronopathy.

The differential diagnosis of sensory neuronopathy is limited, with 4 main possibilities: paraneoplastic, toxic, inflammatory, and inherited. In our patient, the asymmetry at onset, relatively short history, relatively rapid progression, and negative family history make an inherited sensory neuronopathy unlikely. There was no exposure to known dorsal root ganglion toxins such as cis-platinum (1) or megadose pyridoxine (2). Therefore, the patient most likely suffered from a paraneoplastic or an inflammatory sensory neuronopathy.

Sensory neuronopathy may occur in association with several inflammatory or autoimmune diseases, among which the ataxic neuronopathy seen in Sjögren's syndrome is best characterized (3). The hallmark of Sjögren's syndrome is xerophthalmia and xerostomia (sicca syndrome); articular symptoms, inflammatory arthropathy, or frank rheumatoid arthritis may also occur. Sjögren's syndrome sensory neuronopathy presents with areflexia, gait impairment, and prominent loss of proprioception. Biopsies of dorsal root ganglia have shown T-cell lymphocyte invasion with loss of sensory neurons (3). Several other immune-inflammatory conditions have been associated with sensory neuronopathy, including primary biliary cirrhosis, viral hepatitis, and benign monoclonal gammopathy $(4,5)$. Acute febrile illnesses have preceded some cases.

Paraneoplastic sensory neuronopathy (PSN, also called subacute sensory neuropathy or carcinomatous sensory neuropathy) was described in 1948 by Denny-Brown (6) and was one of the key early observations which lead to the concept of "remote effects of cancer" (i.e., nonmetastatic) on the nervous system. Most cases of PSN are now known to be associated with small cell lung cancer (7), although breast and other cancers sometimes are found. Importantly, the underlying cancers are often occult, and the neurological symptoms usually precede symptoms of the cancer. In one series, the mean interval between onset of the neuropathic symptoms and diagnosis of cancer was 5 months (7); intervals up to 7 years before discovery of the cancer have been reported (8).

PSN, like most neurological paraneoplastic syndromes, is thought to be an immune-mediated process, a byproduct of the host immune response to the neoplasm. In the mid-1980s, patients with PSN and small cell lung cancer were found to have an autoantibody in the serum and cerebrospinal fluid that recognizes small cell lung cancer cells as well as a 35-40 kD protein in the nuclei of neurons (Figure 1) $(9,10)$. Known as antineuronal nuclear type 1 (ANNA-1) or anti-Hu, this antibody has proved to be a specific marker for small cell lung cancer in patients with sensory neuronopathy. ANNA-1 antibodies are not found in sensory neuronopathy patients without cancer (8).

\section{FURTHER INVESTIGATION}

Serum was sent to the Neuroimmunology Laboratory of the Mayo Clinic (Dr. V. Lennon). The ANNA-1 titer was 1:15,360 (normal <1:60); other paraneoplastic autoantibodies (ANNA-2, anti-Purkinje cell, anti-N and anti-P/Q type calcium channels) were absent. The patient was immediately admitted to the Montreal General Hospital, where a chest X-ray suggested a left hilar mass. Bronchoscopic biopsy revealed small cell lung cancer. A cranial CT scan, performed for staging, showed numerous tiny cerebral metastases without mass effect. The patient was treated with chemotherapy and cranial irradiation.

\section{DISCUSSION}


This case illustrates the value of paraneoplastic autoantibodies in alerting the clinician to the presence of an occult tumor in a patient with an undiagnosed neurological syndrome. Several distinctive neurological syndromes may occur as "remote effects" of cancer. Such syndromes are uncommon even in large hospitals. Determining that a patient's neurological syndrome is paraneoplastic can be difficult because identical neurological syndromes may occur in patients without cancer and the underlying cancer is often minute and otherwise asymptomatic. Further diagnostic difficulty occurs with patients whose neurological picture combines elements of two or more of the classical syndromes, a situation that is not uncommon.

Despite these complexities, each paraneoplastic syndrome tends to occur with a particular neoplasm, which helps to focus the workup once a paraneoplastic syndrome is suspected. In addition, while the sometimes small size and local confinement of the tumor may hinder its radiographic detection, these same features improve the oncological prognosis.

At least three varieties of paraneoplastic autoantibodies are known, each typically associated with a particular neurological syndrome (Table 1) (11). ANNA-1 seropositivity, as in this patient, is highly specific for small cell lung cancer. Lucchinetti et al. found small cell lung cancer in 105 of 126 seropositive patients (12), and Dalmau et al. in 55 of 71 (13). Despite the consistent association of ANNA-1 seropositivity with small cell lung cancer, patients who are ANNA-1 positive do not have a uniform neurological syndrome. Sensory neuronopathy is probably most common, but other varieties of peripheral neuropathy, encephalomyelitis, autonomic failure, or even intestinal pseudo-obstruction may dominate the clinical picture.

The pathogenic role of the ANNA-1 antibodies is unclear. It has not been possible to develop an animal model, either by passive transfer of human ANNA-1 antibodies or by immunization with recombinant bacterial proteins (14). Treatments that decrease serum titers of ANNA-1 antibodies have generally had no impact on the neurological syndrome, perhaps because many patients likely have irreversible neuronal loss by the time any treatment is given. Unfortunately, antineoplastic treatments have likewise produced little benefit neurologically, even where an oncological response is achieved (7).

\section{ACKNOWLEDGMENT}

The authors thank Dr. V. Lennon (Mayo Clinic) for performing the ANNA-1 assay, for Figure 1, and for helpful discussions.

\section{REFERENCES}

1. Mollmann JE. Cisplatin neurotoxicity. New England Journal of Medicine 322: 126-27; 1990.

2. Schaumburg H, Kaplan J, Windebank A, et al. Sensory neuropathy from pyridoxine abuse: A new megavitamin syndrome. New England Journal of Medicine 309: 445-8; 1983.

3. Griffin JW, Cornblath DR, Alexander E, et al. Ataxic sensory neuropathy and dorsal root ganglionitis associated with Sjogren's syndrome. Annals of Neurology 27: 304-15; 1990.

4. Windebank AJ, Blexrud MD, Dyck PJ, et al. The syndrome of acute sensory neuropathy: Clinical features and electrophysiologic and pathologic changes. Neurology 40: 584-91; 1990.

5. Dalakas MC. Chronic idiopathic ataxic neuropathy. Annals of Neurology 19: 545-54; 1986.

6. Denny-Brown D. Primary sensory neuropathy with muscular changes associated with carcinoma. Journal of Neurology, Neurosurgery and Psychiatry 11: 73-87; 1948.

7. Chalk CH, Windebank AJ, Kimmel DW, et al. The distinctive clinical features of paraneoplastic sensory 
neuronopathy. Canadian Journal of Neurological Science 19: 346-51; 1992.

8. Chalk CH, Lennon VA, Stevens JC, et al. Seronegativity for type 1 antineuronal nuclear antibodies ("anti$\mathrm{Hu}$ ") in subacute sensory neuronopathy patients without cancer. Neurology 43: 2209-11; 1993.

9. Kiers L, Altermatt HJ, Lennon VA. Paraneoplastic anti-neuronal nuclear IgG autoantibodies (type 1) localize antigen in small cell lung carcinoma. Mayo Clinic Proceedings 66: 1209-16; 1991.

10. Graus F, Cordon-Cardo C, Posner JB. Neuronal antinuclear antibody in sensory neuronopathy from lung cancer. Neurology 35: 538-43; 1985.

11. Lennon VA. Calcium channel and related paraneoplastic disease autoantibodies. In: Peter JB, Shoenfeld Y, eds. Autoantibodies. Amsterdam: Elsevier Science B.V.; 1996: 139-147.

12. Lucchinetti CF, Kimmel DW, Lennon VA. Clinical, oncological, and serological profiles of patients seropositive for type 1 anti-neuronal nuclear antibody (ANNA-1, a.k.a. "anti-Hu"). Neurology 44 (Suppl 2): A156.(abstract); 1994.

13. Dalmau J, Graus F, Rosenblum MK, et al. Anti-Hu-associated paraneoplastic encephalomyelitis/sensory neuronopathy: A clinical study of 71 patients. Medicine 71: 59-72; 1992.

14. Sillevis Smitt PAE, Manley GT, Posner JB. Immunization with the paraneoplastic encephalomyelitis antigen $\mathrm{HuD}$ does not cause neurologic disease in mice. Neurology 46: 1873-78; 1995.

15. Peterson K, Rosenblum MK, Kotanides H, et al. Paraneoplastic cerebellar degeneration: I. A clinical analysis of 55 anti-Yo antibody-positive patients. Neurology 42: 1931-37; 1992.

16. O'Neill JH, Murray NMF, Newsom-Davis J. The Lambert-Eaton syndrome: A review of 50 cases. Brain 111: 577-96; 1988.

17. Anderson NE, Budde-Steffen C, Rosenblum MK, et al. Opsoclonus, myoclonus, ataxia, and encephalopathy in adults with cancer: A distinct paraneoplastic syndrome. Medicine 67: 100-9; 1988.

\section{BIOGRAPHY}

Theodore Wein, M.D., received his M.D. at the University of Vermont (Burlington, Vermont, USA) in 1993 and is currently in his final year of neurology residency at McGill University (Montreal, Quebec, Canada). Colin Chalk, M.D.c.M., F.R.C.P.(C), (McGill University, 1984) is Assistant Professor, Department of Neurology and Neurosurgery, McGill University and Associate Physician, Department of Medicine, Montreal General Hospital. He trained in neurology at Oxford University (Oxford, England) and in neurology and peripheral nerve diseases at Mayo Clinic (Rochester, Minnesota, USA). He is also Director of the Neurology Residency Training Programme at McGill University.

Copyright (C) 1996 by MJM 